\title{
Challenges in nuclear medicine: innovative theranostic tools for personalized medicine
}

\section{Françoise Kraeber-Bodéré* and Jacques Barbet}

Nuclear Medicine, University Hospital-ICO, CRCNA, INSERM U892, CNRS UMR 6299, Nantes and GIP Arronax, Saint-Herblain, France

${ }^{*}$ Correspondence: francoise.bodere@chu-nantes.fr

Edited and reviewed by:

Otto C. Boerman, Radboud University Medical Center, Netherlands

Keywords: nuclear medicine, personalized medicine, PET imaging, FDG-PET, targeted therapies

Over the past few years, nuclear medicine has undergone impressive growth with the development of positron emission tomography (PET), especially using ${ }^{18} \mathrm{~F}$ fluoro-deoxy-glucose $\left({ }^{18} \mathrm{FDG}\right)$, and new approaches in targeted radionuclide therapy. These developments pave the way for personalized medicine by offering practical solutions, especially in oncology, neurology, and cardiology. Novel radiopharmaceuticals targeting relevant biomarkers are powerful patient selection tools for patients who may benefit from targeted therapies, and for early therapeutic response assessment. Moreover, once labeled with beta- or alpha-emitters, radiopharmaceuticals targeting relevant molecular markers expressed by different solid tumors, and hemopathies can be used for radionuclide therapy. The final objective here is to eradicate residual cancer disease by using cytotoxic mechanisms complementary to those of "non-radioactive" therapies. PET imaging and targeted radionuclide therapy then come together in the context of the theranostic approach to adapt injected activity for personalized therapy.

${ }^{18}$ FDG-PET demonstrates the high accuracy and the clinical benefits of noninvasive whole-body imaging. It is used in clinical practice for initial staging and therapy evaluation in several solid tumors and hemopathies. ${ }^{18}$ FDG-PET is also applied outside oncology to explore dementia, assess myocardial viability, or detect infectious and inflammatory processes. However, ${ }^{18}$ FDG is not a specific tracer. For example, in solid tumor or lymphoma assessment, ${ }^{18}$ FDG-PET does not distinguish tumors from inflammation, inducing false positive results, especially after therapies inducing inflammatory or immune reactions. New radiopharmaceuticals are needed to better characterize pathologic processes and to predict and assess response to therapy (1).

In oncology, the development of ${ }^{18}$ FDG-PET is ongoing, particularly for therapy response assessment. Image acquisition and analysis protocols are being further standardized to improve diagnostic accuracy (2). For example, specific criteria have been elaborated to assess lymphoma or solid tumor response to therapy (2-4). For tumors with low avidity for ${ }^{18} \mathrm{FDG}$, other ${ }^{18} \mathrm{~F}$-labeled compounds are being proposed (e.g., ${ }^{18} \mathrm{~F}$-choline in prostate cancer or hepatocellular carcinoma) (5). In addition, phenotype-specific tracers are needed for theranostic applications: ${ }^{68} \mathrm{Ga}$-labeled somatostatin analogues improve image quality in neuroendocrine tumors in comparison to ${ }^{111}$ In-octreotide available in clinical practice, with a significant clinical impact. Several ${ }^{68}$ Ga-labeled somatostatin analogues with variable performances have been evaluated (6-8). These novel imaging radiopharmaceuticals are particularly interesting because cold- and radio-labeled somatostatin analogues are efficient for therapy of neuroendocrine tumors. Numerous peptides, targeting other receptors, are in development. Radiolabeled bombesin analogues show promise for prostate cancer $(9,10)$. Radiolabeled RDG peptides targeting the $\alpha_{v} \beta_{3}$ integrin have potential in a large spectrum of indications in the field of oncology but also in cardiovascular diseases $(11,12)$.

Targeted therapies, including monoclonal antibodies (MAbs), experience a considerable growth in cancer management. MAbs are also promising vectors for theranostic approaches, to better identify patients who will respond and to monitor responses (13). Based on immuno-PET, treatment strategies could be tailored for individual patients before administering expensive and potentially toxic therapies. Until now, only invasive methods such as biopsy with immunohistochemistry analysis could identify patients who have the highest chance of response to antibodybased therapy. Immuno-PET can offer a non-invasive solution to quantitatively assess target expression. For example, antiHER2 therapeutic agents are most effective in patients who have HER2-positive breast cancer as determined by immunohistochemistry. It has been proven that MAbs labeled with ${ }^{68} \mathrm{Ga},{ }^{64} \mathrm{Cu}$, or ${ }^{89} \mathrm{Zr}$ could noninvasively identify lesions that are likely to respond to therapy (14-16). Immuno-PET is also a powerful innovation to improve knowledge about the efficacy and in vivo behavior of MAbs.

Imaging plays an increasing role in the development of new drugs by pharmaceutical companies: in vivo imaging constitutes an effective solution for the rapid assessment of drug candidates, which may be radiolabeled to monitor their pharmacokinetics and biodistribution during preclinical and early clinical phases. Alternatively, molecular imaging using radiopharmaceuticals, combined with biomarkers, gives information about the quantitative variation of molecular targets during treatments. Indeed, if ${ }^{18} \mathrm{FDG}-\mathrm{PET}$ can be used (17), radiolabeled tyrosine kinase inhibitors (TKI) analogues are also developed to evaluate TKI efficacy in clinical trials by PET imaging (18).

Nuclear medicine is also advancing cancer therapy. Clear examples are the treatment of non-Hodgkin's lymphoma by antiCD20 or CD22 MAbs labeled with yttrium$90(19,20)$ and of neuroendocrine tumors by somatostatin analogues labeled with 
yttrium-90 or lutetium-177 $(21,22)$. However, many other tumors must be addressed and promising results have been reported in prostate cancer and colorectal carcinoma $(23,24)$. These treatments are generally not curative. More efficient radiopharmaceuticals and more toxic radionuclides, such as alpha-emitters, as well as combination therapy or multiple injection protocols, seldom studied yet in the field of targeted radionuclide therapy, should be developed with the objective of killing the last tumor cell, which is most probably a cancer stem cell (25). Efficacy of alpha-therapy has been recently shown in a pre-clinical model of multiple myeloma using anti-syndecan 1 MAbs labeled with the alpha-emitter ${ }^{213} \mathrm{Bi}$ (26). Fractionated radioimmunotherapy with ${ }^{90}$ Y-clivatuzumab and low-dose gemcitabine demonstrated activity in advanced pancreatic cancer in a phase I clinical trial (27). Pre-targeting using bispecific antibodies and radiolabeled bivalent haptenpeptides showed promising results in CEApositive tumors $(28,29)$. Predictive patient dosimetry can be obtained using a test dose to adapt injected therapeutic activity to each patient pharmacokinetics and biodistribution (30).

The development of new radiopharmaceuticals requires radionuclides with physical half-lives that match the vector biological half-lives. For imaging purposes, short half-life PET emitters such as ${ }^{68} \mathrm{Ga}$ and ${ }^{18} \mathrm{~F}$ are well suited for labeling drugs with fast distribution kinetics. The short half-life reduces exposure and allows for pharmacological studies of receptor occupancy, for instance. For other vectors that reach their target more slowly, such as antibodies, radionuclides with longer halflives are needed $\left({ }^{64} \mathrm{Cu},{ }^{44 / 44 m} \mathrm{Sc},{ }^{89} \mathrm{Zr}\right)$ (31). In addition, couples of $\beta^{+} / \beta^{-}$isotopes (e.g., ${ }^{64} \mathrm{Cu}$ and ${ }^{67} \mathrm{Cu}$ ) are of particular interest for PET/therapy radiopharmaceuticals. For therapeutic purposes, the penetration path-length of the radioactive emission should match the size of the targeted tumor. Yttrium-90, with its long-range beta ${ }^{-}$emission, is better suited to bulky diseases. However, promising results have been observed using ${ }^{90} \mathrm{Y}-$ radioimmunotherapy in the consolidation setting in responding lymphoma patients (partial or complete responders) after induction therapy (32). Radionuclide such as ${ }^{131} \mathrm{I},{ }^{177} \mathrm{Lu}$, or ${ }^{67} \mathrm{Cu}$ with shorter-range beta $^{-}$emissions should be more favorable in the minimal residual disease setting. Alpha particle emitters offer the theoretical possibility to kill isolated tumor cells and microscopic clusters of tumor cells because of the short tracks of alpha particles (25). For alpha-emitters, ${ }^{213} \mathrm{Bi}$ is available through a ${ }^{225} \mathrm{Ac} /{ }^{213} \mathrm{Bi}$ generator. Its short half-life $(46 \mathrm{~min})$ and cost are potential limitations. Despite its complex chemistry, ${ }^{211}$ At may be a better candidate for alpha-therapy due to its longer half-life $(7.2 \mathrm{~h})$ and other radionuclides with complex radioactive decay schemes are proposed $\left({ }^{212} \mathrm{~Pb},{ }^{225} \mathrm{Ac},{ }^{227} \mathrm{Th}\right)(33)$.

In the decades to come, we anticipate that new radiopharmaceuticals targeting specific biomarkers will be developed for early diagnosis and prognostic prediction, and to adapt therapies to each patient in oncology, as briefly described above, but also in other domains, such as neurology or cardiology. We believe that PET represents a promising tool for molecular diagnosis, providing non-invasive whole-body cartography of biomarkers, complementary to biological testing. Moreover, we believe that targeted radionuclide therapy will develop, especially in multimodality strategies.

The goal of Nuclear Medicine is to be a forum for publication of pre-clinical and clinical findings that assess the role of new and existing radiopharmaceuticals in the molecular characterization of disease before and after treatment, with the ultimate goal of personalized patient management. This section is also open to contributions in the fields of radiopharmaceutical discovery, new radionuclide therapy procedures that use innovative radionuclides or radiopharmaceuticals, multimodality strategies and original methods of image analysis, quantification, and dosimetric evaluation.

\section{ACKNOWLEDGMENTS}

This work has been supported in part by grants from the French National Agency for Research called "Investissements d'Avenir" Labex IRON $n^{\circ}$ ANR-11-LABX-0018-01 and Equipex ArronaxPlus $n^{\circ}$ ANR-11EQPX-0004 and by a grant from the Pays de la Loire Regional Council called "NucSan."

\section{REFERENCES}

1. Zhao B, Schwartz LH, Larson SM. Imaging surrogates of tumor response to therapy: anatomic and functional biomarkers. J Nucl Med (2009) 50:239-49. doi:10.2967/jnumed.108.056655

2. Wahl RL, Jacene H, Kasamon Y, Lodge MA. From RECIST to PERCIST: evolving considerations for PET response criteria in solid tumors. J Nucl Med (2009) 50(Suppl 1):122S-50S. doi:10.2967/ jnumed.108.057307

3. Juweid ME, Stroobants S, Hoekstra OS, Mottaghy FM, Dietlein M, Guermazi A, et al. Imaging subcommittee of international harmonization project in lymphoma. Use of positron emission tomography for response assessment of lymphoma: consensus of the imaging subcommittee of international harmonization project in lymphoma. J Clin Oncol (2007) 25:571-8. doi:10.1200/JCO.2006.08.2305

4. Meignan M, Barrington S, Itti E, Gallamini A, Haioun C, Polliack A. Report on the 4th international workshop on positron emission tomography in lymphoma held in Menton, France, 35 October 2012. Leuk Lymphoma (2014) 55:31-7. doi:10.3109/10428194.2013.802784

5. Apolo AB, Pandit-Taskar N, Morris MJ. Novel tracers and their development for the imaging of metastatic prostate cancer. J Nucl Med (2008) 49:2031-41. doi:10.2967/jnumed.108.050658

6. Gabriel M, Decristoforo C, Kendler D, Dobrozemsky G, Heute D, Uprimny C, et al. 68Ga-DOTATyr3-octreotide PET in neuroendocrine tumors: comparison with somatostatin receptor scintigraphy and CT. J Nucl Med (2007) 48:508-18. doi:10.2967/jnumed.106.035667

7. Ambrosini V, Fani M, Fanti S, Forrer F, Maecke HR. Radiopeptide imaging and therapy in Europe. J Nucl Med (2011) 52(Suppl 2):42S-55S. doi:10. 2967/jnumed.110.085753

8. Wild D, Bomanji JB, Benkert P, Maecke $\mathrm{H}$, Ell PJ, Reubi JC, et al. Comparison of 68Ga-DOTANOC and 68Ga-DOTATATE PET/CT within patients with gastroenteropancreatic neuroendocrine tumors. J Nucl Med (2013) 54:364-72. doi:10.2967/jnumed.112.111724

9. Wild D, Frischknecht M, Zhang H, Morgenstern A, Bruchertseifer F, Boisclair J, et al. Alpha- versus beta-particle radiopeptide therapy in a human prostate cancer model (213Bi-DOTA-PESIN and 213Bi-AMBA versus 177Lu-DOTA-PESIN). Cancer Res (2011) 71:1009-18. doi:10.1158/00085472.CAN-10-1186

10. Liu Y, Hu X, Liu H, Bu L, Ma X, Cheng K, et al. A comparative study of radiolabeled bombesin analogs for the PET imaging of prostate cancer. J Nucl Med (2013) 54:2132-238. doi:10.2967/ jnumed.113.121533

11. Wadas TJ, Deng H, Sprague JE, Zheleznyak A, Weilbaecher KN, Anderson CJ. Targeting the alphavbeta3 integrin for small-animal PET/CT of osteolytic bone metastases. J Nucl Med (2009) 50:1873-80. doi:10.2967/jnumed.109.067140

12. Beer AJ, Pelisek J, Heider P, Saraste A, Reeps C, Metz S, et al. PET/CT imaging of integrin $\alpha v \beta 3$ expression in human carotid atherosclerosis. JACC Cardiovasc Imaging (2014) 7:178-87. doi:10.1016/ j.jcmg.2013.12.003

13. Boerman OC, Oyen WJ. Immuno-PET of cancer: a revival of antibody imaging. J Nucl Med (2011) 52:1171-2. doi:10.2967/jnumed.111.089771

14. Dijkers EC, Kosterink JG, Rademaker AP, Perk LR, van Dongen GA, Bart J, et al. Development and characterization of clinical-grade 
89Zr-trastuzumab for HER2/neu immunoPET imaging. J Nucl Med (2009) 50:974-81. doi:10. 2967/jnumed.108.060392

15. Baum RP, Prasad V, Müller D, Schuchardt C, Orlova A, Wennborg A, et al. Molecular imaging of HER2-expressing malignant tumors in breast cancer patients using synthetic $111 \mathrm{In}$ - or $68 \mathrm{Ga}$-labeled affibody molecules. J Nucl Med (2010) 51:892-7. doi:10.2967/jnumed.109.073239

16. Tamura K, Kurihara H, Yonemori K, Tsuda H, Suzuki J, Kono Y, et al. 64Cu-DOTA-trastuzumab PET imaging in patients with HER2-positive breast cancer. J Nucl Med (2013) 54:1869-75. doi:10. 2967/jnumed.112.118612

17. Kraeber-Bodéré F, Carlier T, Naegelen VM, Shochat E, Lumbroso J, Trampal C, et al. Differences in the biologic activity of 2 novel MEK inhibitors revealed by 18 F-FDG PET: analysis of imaging data from 2 phase I trials. J Nucl Med (2012) 53:1836-46. doi:10.2967/jnumed.112.109421

18. van Dongen GA, Poot AJ, Vugts DJ. PET imaging with radiolabeled antibodies and tyrosine kinase inhibitors: immuno-PET and TKI-PET. Tumour Biol (2012) 33:607-15. doi:10.1007/s13277-0120316-4

19. Morschhauser F, Kraeber-Bodéré F, Wegener WA, Harousseau JL, Petillon MO, Huglo D, et al. High rates of durable responses with anti-CD22 fractionated radioimmunotherapy: results of a multicenter, phase I/II study in non-Hodgkin's lymphoma. J Clin Oncol (2010) 28:3709-16. doi:10. 1200/JCO.2009.27.7863

20. Illidge TM, Mayes S, Pettengell R, Bates AT, Bayne M, Radford JA, et al. Fractionated ${ }^{90} \mathrm{Y}$ ibritumomab tiuxetan radioimmunotherapy as an initial therapy of follicular lymphoma: an international phase II study in patients requiring treatment according to GELF/BNLI criteria. J Clin Oncol (2014) 32:212-8. doi:10.1200/JCO.2013.50. 3110

21. Kwekkeboom DJ, de Herder WW, Kam BL, van Eijck $\mathrm{CH}$, van Essen M, Kooij PP, et al. Treatment with the radiolabeled somatostatin analog [177 Lu-DOTA 0,Tyr3] octreotate: toxicity, efficacy, and survival. J Clin Oncol (2008) 26:2124-30. doi:10.1200/JCO.2007.15.2553

22. Bodei L, Mueller-Brand J, Baum RP, Pavel ME, Hörsch D, O'Dorisio MS, et al. The joint IAEA, EANM, and SNMMI practical guidance on peptide receptor radionuclide therapy (PRRNT) in neuroendocrine tumours. Eur J Nucl Med $\mathrm{Mol}$ Imaging (2013) 40:800-16. doi:10.1007/s00259012-2330-6

23. Tagawa ST, Beltran H, Vallabhajosula S, Goldsmith SJ, Osborne J, Matulich D, et al. Anti-prostatespecific membrane antigen-based radioimmunotherapy for prostate cancer. Cancer (2010) 116:1075-83. doi:10.1002/cncr.24795

24. Liersch T, Meller J, Bittrich M, Kulle B, Becker $\mathrm{H}$, Goldenberg DM. Update of carcinoembryonic antigen radioimmunotherapy with 131Ilabetuzumab after salvage resection of colorectal liver metastases: comparison of outcome to a contemporaneous control group. Ann Surg Oncol (2007) 14:2577-90. doi:10.1245/s10434006-9328-x

25. Barbet J, Chérel M, Chatal JF. Alpha particles more promising than toxins? Eur J Nucl Med Mol Imaging (2010) 37:849-50. doi:10.1007/s00259010-1421-5

26. Chérel M, Gouard S, Gaschet J, Saï-Maurel C, Bruchertseifer F, Morgenstern A, et al. 213Bi radioimmunotherapy with an anti-mCD138 monoclonal antibody in a murine model of multiple myeloma. J Nucl Med (2013) 54:1597-604. doi:10.2967/jnumed.112.111997

27. Ocean AJ, Pennington KL, Guarino MJ, Sheikh A, Bekaii-Saab T, Serafini AN, et al. Fractionated radioimmunotherapy with (90) Y-clivatuzumab tetraxetan and low-dose gemcitabine is active in advanced pancreatic cancer: a phase 1 trial. Cancer (2012) 118:5497-506. doi:10.1002/cncr.27592

28. Chatal JF, Campion L, Kraeber-Bodéré F, Bardet S, Vuillez JP, Charbonnel B, et al. Survival improvement in patients with medullary thyroid carcinoma who undergo pretargeted anticarcinoembryonic-antigen radioimmunotherapy: a collaborative study with the French Endocrine Tumor Group. J Clin Oncol (2006) 24:1705-11. doi:10.1200/JCO.2005.04.4917

29. Salaun PY, Campion L, Bournaud C, FaivreChauvet A, Vuillez JP, Taieb D, et al. Phase II trial of anticarcinoembryonic antigen pretargeted radioimmunotherapy in progressive metastatic medullary thyroid carcinoma: biomarker response and survival improvement. J Nucl Med (2012) 53:1185-92. doi:10.2967/jnumed.111.101865
30. Schoffelen R, Woliner-van der Weg W, Visser EP, Goldenberg DM, Sharkey RM, McBride WJ, et al. Predictive patient-specific dosimetry and individualized dosing of pretargeted radioimmunotherapy in patients with advanced colorectal cancer. Eur J Nucl Med Mol Imaging (2014). doi:10.1007/ s00259-014-2742-6

31. Wu AM. Antibodies and antimatter: the resurgence of immuno-PET. J Nucl Med (2009) 50:2-5. doi:10.2967/jnumed.108.056887

32. Morschhauser F, Radford J, Van Hoof A, Botto B, Rohatiner AZ, Salles G, et al. 90Yttriumibritumomab tiuxetan consolidation of first remission in advanced-stage follicular non-Hodgkin lymphoma: updated results after a median followup of 7.3 years from the international, randomized, phase III first-LineIndolent trial. J Clin Oncol (2013) 31:1977-83. doi:10.1200/JCO.2012. 45.6400

33. Huclier-Markai S, Alliot C, Varmenot N, Cutler CS, Barbet J. Alpha-emitters for immuno-therapy: a review of recent developments from chemistry to clinics. Curr Top Med Chem (2012) 12:2642-54. doi:10.2174/1568026611212230002

Conflict of Interest Statement: The authors declare that the research was conducted in the absence of any commercial or financial relationships that could be construed as a potential conflict of interest.

Received:05 May 2014; accepted:04 June 2014; published online: 03 July 2014.

Citation: Kraeber-Bodéré F and Barbet J (2014) Challenges in nuclear medicine: innovative theranostic tools for personalized medicine. Front. Med. 1:16. doi: $10.3389 /$ fmed.2014.00016

This article was submitted to Nuclear Medicine, a section of the journal Frontiers in Medicine.

Copyright (c) 2014 Kraeber-Bodéré and Barbet. This is an open-access article distributed under the terms of the Creative Commons Attribution License (CC BY). The use, distribution or reproduction in other forums is permitted, provided the original author(s) or licensor are credited and that the original publication in this journal is cited, in accordance with accepted academic practice. No use, distribution or reproduction is permitted which does not comply with these terms. 